\title{
Fundamental Spectral Theory of Fractional Singular Sturm-Liouville Operator
}

\author{
Erdal Bas \\ Department of Mathematics, Faculty of Science, Firat University, 23119 Elazig, Turkey \\ Correspondence should be addressed to Erdal Bas; erdalmat@yahoo.com
}

Received 29 May 2013; Accepted 19 July 2013

Academic Editor: Kehe Zhu

Copyright (C) 2013 Erdal Bas. This is an open access article distributed under the Creative Commons Attribution License, which permits unrestricted use, distribution, and reproduction in any medium, provided the original work is properly cited.

We give the theory of spectral properties for eigenvalues and eigenfunctions of Bessel type of fractional singular Sturm-Liouville problem. We show that the eigenvalues and eigenfunctions of the problem are real and orthogonal, respectively. Furthermore, we prove new approximations about the topic.

\section{Introduction}

Sturm-Liouville problem was first developed in a number of papers that were published by these authors in 1836 and 1837. Charles-François Sturm (1803-1855), Professor of Mechanics at the Sorbonne, had been interested, since about 1833, in the problem of heat flow in bars, so he was well aware of eigenvalue-type problems. He worked closely with his friend Joseph Liouville (1809-1882), Professor of Mathematics at the Collège de France, on the general properties of second-order differential equations. Liouville also made many contributions to the general field of analysis, see [1].

A Sturm-Liouville boundary value problem consists of a second order linear ordinary differential equation

$$
-\left(p y^{\prime}\right)^{\prime}+q y=\lambda w y, \quad(a, b)
$$

and boundary conditions. Here $(a, b)$ is a bounded or unbounded open interval of the real line $R$. The coefficients $p, q, w:(a, b)$ into $R ; \lambda \in \mathbb{C}$, the complex field. Spectral analysis finds applications in many diverse fields. Mathematical techniques could be developed into a more suitable and significant course by presenting them within the more general Sturm-Liouville theory in $L_{2}$. The SturmLiouville problems are important in many areas of science, engineering and mathematics. It is known that the spectral characteristics are spectra, spectral functions, scattering data, norming constants, etc. According to the theory a linear second-order differential operator which is self-adjoint has an orthogonal sequence of eigenfunctions in $L_{2}$. Spectral properties of Sturm-Liouville operators are often derived, directly or indirectly, as a consequence of an established link between large distance asymptotic behavior of solutions of the associated differential equation and spectral properties of the corresponding differential operator. Sturm-Liouville problems are divided into regular and singular types. Differential equations such as Bessel, hydrogen atom, Hermitte, Jakobi, and Legendre equations can be transformed into Sturm-Liouville equations. There are many studies on these issues [2-7]. We also discuss the radial part of Schrödinger's equation for the Bessel equation.

Fractional calculus is "the theory of derivatives and integrals of any arbitrary real or complex order, which unify and generalize the notions of integer-order differentiation and $n$-fold integration" [6-13]. In recent years, the concept of fractional calculus, originated from Leibniz, has achieved increasing interest during the last two decades. In particular, the last decade has scientific papers concerning fractional quantum mechanics. It has been proved that many systems in different fields of science and engineering can be modeled more accurately using fractional derivatives [8-17]. Fractional calculus has increasing importance for the last years because fractional calculus has been applied to almost every field of science. They are viscoelasticity, electrical engineering, electrochemistry, biology, biophysics and bioengineering, signal and image processing, mechanics, mechatronics, physics, and control theory. We note that 
ordinary derivatives in a traditional Sturm-Liouville problem are replaced with fractional derivatives, and the resulting problems are solved using some numerical methods [18-23]. Furthermore, Klimek and Argawal [24] define a fractional Sturm-Liouville operator, introduce a regular fractional Sturm-Liouville problem, and investigate the properties of the eigenfunctions and the eigenvalues of the operator. In this paper, our purpose is to introduce singular fractional SturmLiouville problem having Bessel type and prove spectral properties of spectral data for the operator.

Let us give the boundary value problem for Bessel equation and necessary data as follows.

\section{Preliminaries}

Now, consider the following Bessel equation:

$$
\frac{d^{2} y}{d x^{2}}+\left(\lambda-\frac{v^{2}-1 / 4}{x^{2}}\right) y=0
$$

where $\lambda$ and $v$ are real numbers. The Bessel equation for having the analogous singularity is given in [5].

Definition 1 (see [10]). Let $0<\alpha \leq 1$. The left-sided and rightsided Riemann-Liouville integrals of order $\alpha$, respectively, are given by the formulas

$$
\begin{aligned}
& \left(I_{a,+}^{\alpha} f\right)(x)=\frac{1}{\Gamma(\alpha)} \int_{a}^{x}(x-s)^{\alpha-1} f(s) d s, \quad x>a, \\
& \left(I_{b,-}^{\alpha} f\right)(x)=\frac{1}{\Gamma(\alpha)} \int_{x}^{b}(s-x)^{\alpha-1} f(s) d s, \quad x<b,
\end{aligned}
$$

where $\Gamma$ denotes the gamma function.

Definition 2 (see [10]). Let $0<\alpha \leq 1$. The left-sided and right-sided Riemann-Liouville derivatives of order $\alpha$, respectively, are defined as follows:

$$
\begin{gathered}
\left(D_{a,+}^{\alpha} f\right)(x)=D\left(I_{a,+}^{1-\alpha} f\right)(x) \quad x>a, \\
\left(D_{b,-}^{\alpha} f\right)(x)=-D\left(I_{b,-}^{1-\alpha} f\right)(x) \quad x<b .
\end{gathered}
$$

Analogous formulas yield the left-sided and right-sided Caputo derivatives of order $\alpha$ :

$$
\begin{aligned}
& \left({ }^{C} D_{a,+}^{\alpha} f\right)(x)=\left(I_{a,+}^{1-\alpha} D f\right)(x) \quad x>a, 0<\alpha \leq 1 \\
& \left({ }^{C} D_{b,-}^{\alpha} f\right)(x) \\
& =\left(I_{b,-}^{1-\alpha}(-D) f\right)(x) \quad x<b, 0<\alpha \leq 1 .
\end{aligned}
$$

Definition 3 (see [14]). The general function ${ }_{p} \Psi_{q}(z)$ is defined for $z \in \mathbb{C}, a_{l}, b_{j} \in \mathbb{C}$, and $\alpha_{l}, \beta_{j} \in \mathbb{R}(l=1, \ldots, p ; j=$ $1, \ldots, q)$ by the series

$$
{ }_{p} \Psi_{q}(z)={ }_{p} \Psi_{q}\left[\begin{array}{c}
\left(a_{1}, \alpha_{1}\right)_{1, p} \\
\left(b_{1}, \beta_{1}\right)_{1, q}
\end{array} \mid z\right]=\sum_{k=0}^{\infty} \frac{\prod_{l=1}^{p} \Gamma\left(a_{l}+\alpha_{l} k\right)}{\prod_{j=1}^{q} \Gamma\left(b_{j}+\beta_{j} k\right)} \frac{z^{k}}{k !} .
$$

This general Wright function was investigated by Fox who presented its asymptotic expansion for large values of the argument $z$ under the condition

$$
\sum_{j=1}^{q} \beta_{j}-\sum_{l=1}^{p} \alpha_{l}>1
$$

If these conditions are satisfied, the series in (6) is convergent for any $z \in \mathbb{C}$.

Theorem 4 (see [14]). Let $a_{l}, b_{j} \in \mathbb{C}$, and $\alpha_{l}, \beta_{j} \in \mathbb{R}(l=$ $1, \ldots, p ; j=1, \ldots q)$, and let

$$
\begin{gathered}
\Delta=\sum_{j=1}^{q} \beta_{j}-\sum_{l=1}^{p} \alpha_{l}, \\
\delta=\prod_{l=1}^{p}\left|\alpha_{l}\right|^{-\alpha_{l}} \prod_{j=1}^{q}\left|\beta_{j}\right|^{\beta_{j}}, \\
\mu=\sum_{j=1}^{q} b_{j}-\sum_{l=1}^{p} a_{l}+\frac{p-q}{2} .
\end{gathered}
$$

(i) If $\Delta>-1$, then the series in (6) is absolutely convergent for all $z \in \mathbb{C}$.

(ii) If $\Delta=-1$, then the series in (6) is absolutely convergent for $|z|<\delta$ and for $|z|=\delta$ and $\mathfrak{R}(\mu)>1 / 2$.

Property 1. The fractional differential operators defined in(4)-(5) satisfy the following identities:

(i)

$$
\begin{aligned}
& \int_{a}^{b} f(x) D_{b,-}^{\alpha} g(x) d x \\
& \quad=\int_{a}^{b} g(x){ }^{C} D_{a,+}^{\alpha} f(x) d x-\left.f(x) I_{b,-}^{1-\alpha} g(x)\right|_{a} ^{b}
\end{aligned}
$$

(ii)

$$
\begin{aligned}
\int_{a}^{b} f(x) D_{b,-}^{\alpha} g(x){ }^{C} D_{a,+}^{\alpha} k(x) d x \\
=\int_{a}^{b} g(x){ }^{C} D_{a,+}^{\alpha} f(x){ }^{C} D_{a,+}^{\alpha} k(x) d x \\
\quad-\left.f(x) I_{b,-}^{1-\alpha} g(x){ }^{C} D_{a,+}^{\alpha} k(x)\right|_{a} ^{b}
\end{aligned}
$$

(iii)

$$
\begin{aligned}
\int_{a}^{b} f(x) D_{a,+}^{\alpha} g(x) d x \\
\quad=\int_{a}^{b} g(x){ }^{C} D_{b,-}^{\alpha} f(x) d x+\left.f(x) I_{a,+}^{1-\alpha} g(x)\right|_{a} ^{b}
\end{aligned}
$$


Property 2 (see [24]). Assume that $\alpha \in(0,1), \beta>\alpha$, and $f \in C[a, b]$. Then the relations

$$
\begin{gathered}
D_{a,+}^{\alpha} I_{a,+}^{\alpha} f(x)=f(x), \\
D_{b,-}^{\alpha} I_{b,-}^{\alpha} f(x)=f(x), \\
D_{a,+}^{\alpha} I_{a,+}^{\beta} f(x)=I_{a,+}^{\beta-\alpha} f(x), \\
D_{b,-}^{\alpha} I_{b,-}^{\beta} f(x)=I_{b,-}^{\beta-\alpha} f(x), \\
{ }^{C} D_{a,+}^{\alpha} I_{a,+}^{\alpha} f(x)=f(x), \\
{ }^{C} D_{b,-}^{\alpha} I_{b,-}^{\alpha} f(x)=f(x),
\end{gathered}
$$

hold for any $x \in[a, b]$. Furthermore, the integral operators defined in (3) satisfy the following semigroup properties:

$$
I_{a,+}^{\alpha} I_{a,+}^{\beta}=I_{a,+}^{\alpha+\beta}, \quad I_{b,-}^{\alpha} I_{b,-}^{\beta}=I_{b,-}^{\alpha+\beta} .
$$

Now, let us take up a singular fractional boundary problem for Bessel operator and give some spectral results.

\section{Main Results}

3.1. A Singular Fractional Sturm-Liouville Problem for Bessel Operator. Fractional Sturm-Liouville problem for Bessel operator denotes the differential part containing the left- and right-sided derivatives. Let us use the form of the integration by parts formulas (10), (11) for this new approximation. Properties of eigenfunctions and eigenvalues in the theory of classical Sturm-Liouville problems are related to the integration by parts formula for the first-order derivatives. In the corresponding fractional version, we note that both left and right derivatives appear and the essential pairs are the left Riemann-Liouville derivative with the right Caputo derivative and the right Riemann-Liouville derivative with the left Caputo one. Spectral properties of Sturm-Liouville operators are often derived, directly or indirectly, as a consequence of an established link between large distance asymptotic behavior of solutions of the associated differential equation and spectral properties of the corresponding Bessel operator.

Definition 5. Let $\alpha \in(0,1)$. Fractional Bessel operator is written as

$$
\mathscr{L}_{\alpha[B]}=D_{1,-}^{\alpha} p(x)^{C} D_{0,+}^{\alpha}+\left(q(x)-\frac{v^{2}-1 / 4}{x^{2}}\right) .
$$

Considering the fractional Bessel equation

$$
\mathscr{L}_{\alpha[B]} y_{\lambda}(x)+\lambda w_{\alpha}(x) y_{\lambda}(x)=0,
$$

where $p(x) \neq 0, w_{\alpha}(x)>0$, for all $x \in(0,1], w_{\alpha}(x)$ is weight function, and $p, q$ are real valued continuous functions in interval $(0,1]$.
The boundary conditions for the operator $\mathscr{L}$ are the following:

$$
\begin{gathered}
y_{\lambda}(0)=0, \\
d_{1} y(1)+d_{2} I_{1,-}^{1-\alpha} p(1){ }^{C} D_{0,+}^{\alpha} y(1)=0,
\end{gathered}
$$

where $d_{1}^{2}+d_{2}^{2} \neq 0$. The fractional boundary-value problem (15)-(16) is fractional Sturm-Liouville problem for Bessel operator.

Theorem 6. Fractional Bessel operator is self-adjoint on $(0,1]$.

Proof. Let us consider the following equation:

$$
\begin{aligned}
\left\langle\mathscr{L}_{\alpha[B]} \varphi, \phi\right\rangle= & \int_{0}^{1} \mathscr{L}_{\alpha[B]} \varphi(x) \cdot \phi(x) d x \\
= & \int_{0}^{1} \phi(x)\left[D_{1,-}^{\alpha} p(x){ }^{C} D_{0,+}^{\alpha} \varphi(x)\right. \\
& \left.+\left(q(x)-\frac{v^{2}-1 / 4}{x^{2}}\right) \varphi(x)\right] d x \\
= & \int_{0}^{1} \phi(x) D_{1,-}^{\alpha} p(x){ }^{C} D_{0,+}^{\alpha} \varphi(x) d x \\
& +\int_{0}^{1}\left(q(x)-\frac{v^{2}-1 / 4}{x^{2}}\right) \varphi(x) \phi(x) d x .
\end{aligned}
$$

By means of equality (10) and boundary conditions (16), we obtain the identity

$$
\begin{aligned}
\left\langle\mathscr{L}_{\alpha[B]} \varphi, \phi\right\rangle= & \int_{0}^{1} p(x){ }^{C} D_{0,+}^{\alpha} \phi(x){ }^{C} D_{0,+}^{\alpha} \varphi(x) d x \\
& -\left.\phi(x) I_{1,-}^{1-\alpha} p(x){ }^{C} D_{0,+}^{\alpha} \varphi(x)\right|_{0} ^{1} \\
& +\int_{0}^{1}\left(q(x)-\frac{v^{2}-1 / 4}{x^{2}}\right) \varphi(x) \phi(x) d x \\
= & \int_{0}^{1} p(x){ }^{C} D_{0,+}^{\alpha} \phi(x){ }^{C} D_{0,+}^{\alpha} \varphi(x) d x \\
& +\frac{d_{1}}{d_{2}} \varphi(1) \phi(1) \\
& +\int_{0}^{1}\left(q(x)-\frac{v^{2}-1 / 4}{x^{2}}\right) \varphi(x) \phi(x) d x .
\end{aligned}
$$


On the other hand, by performing similar operations, we find

$$
\begin{aligned}
\left\langle\varphi, \mathscr{L}_{\alpha[B]} \phi\right\rangle= & \int_{0}^{1} p(x)^{C} D_{0,+}^{\alpha} \varphi(x){ }^{C} D_{0,+}^{\alpha} \phi(x) d x \\
& +\frac{d_{1}}{d_{2}} \varphi(1) \phi(1) \\
& +\int_{0}^{1}\left(q(x)-\frac{v^{2}-1 / 4}{x^{2}}\right) \phi(x) \varphi(x) d x .
\end{aligned}
$$

The right-hand sides of (18) and (19) are equal; hence, we may see that the left sides are equal; that is,

$$
\left\langle\mathscr{L}_{\alpha[B]} \varphi, \phi\right\rangle=\left\langle\varphi, \mathscr{L}_{\alpha[B]} \phi\right\rangle .
$$

Theorem 7. The eigenvalues of fractional Bessel operator (15)(16) are real.

Proof. Let us observe that the following relation results from equality (10):

$$
\begin{aligned}
& \int_{0}^{1} f(x) \mathscr{L}_{\alpha[B]} g(x) d x \\
& =\int_{0}^{1} p(x){ }^{C} D_{0,+}^{\alpha} f(x){ }^{C} D_{0,+}^{\alpha} g(x) d x \\
& \quad-\left.f(x) I_{1,-}^{1-\alpha} p(x){ }^{C} D_{0,+}^{\alpha} g(x)\right|_{0} ^{1} \\
& \quad+\int_{0}^{1}\left(q(x)-\frac{v^{2}-1 / 4}{x^{2}}\right) g(x) f(x) d x .
\end{aligned}
$$

Suppose that $\lambda$ is the eigenvalue for (15)-(16) corresponding to eigenfunction $y$; the following equalities satisfy $y$ and its complex conjugate $\bar{y}$ :

$$
\begin{gathered}
\mathscr{L}_{\alpha[B]} y(x)+\lambda w_{\alpha}(x) y(x)=0, \\
y(0)=0, \\
d_{1} y(1)+d_{2} I_{1,-}^{1-\alpha} p(1)^{C} D_{0,+}^{\alpha} y(1)=0, \\
\mathscr{L}_{\alpha[B]} \bar{y}(x)+\bar{\lambda} w_{\alpha}(x) \bar{y}(x)=0, \\
\bar{y}(0)=0, \\
d_{1} \bar{y}(1)+d_{2} I_{1,-}^{1-\alpha} p(1)^{C} D_{0,+}^{\alpha} \bar{y}(1)=0,
\end{gathered}
$$

where $d_{1}^{2}+d_{2}^{2} \neq 0$. We multiply (22) by function $\bar{y}$ and (24) by function $y$, respectively, and subtract

$$
\begin{aligned}
& (\lambda-\bar{\lambda}) w_{\alpha}(x) y(x) \bar{y}(x) \\
& \quad=y(x) \mathscr{L}_{\alpha[B]} \bar{y}(x)-\bar{y}(x) \mathscr{L}_{\alpha[B]} y(x) .
\end{aligned}
$$

Now, we integrate over interval $(0,1]$, and applying relation (21), and we note that the right-hand side of the integrated equality contains only boundary terms:

$$
\begin{aligned}
& (\lambda-\bar{\lambda}) \int_{0}^{1} w_{\alpha}(x) y(x) \bar{y}(x) d x \\
& =\int_{0}^{1} y(x) \mathscr{L}_{\alpha[B]} \bar{y}(x) d x-\int_{0}^{1} \bar{y}(x) \mathscr{L}_{\alpha[B]} y(x) d x \\
& =\int_{0}^{1} y(x)\left[D_{1,-}^{\alpha} p(x)^{C} D_{0,+}^{\alpha} \bar{y}(x)\right. \\
& \left.\left.+{ }_{0}{ }^{q}(x)-\frac{v^{2}-1 / 4}{x^{2}}\right) \bar{y}(x)\right] d x \\
& +\left.\bar{y}(x) I_{1,-}^{1-\alpha} p(x){ }^{C} D_{0,+}^{\alpha} y(x)\right|_{1} ^{\alpha} p(x){ }^{C} D_{0,+}^{\alpha}(x) y(x) \\
& +\left.\bar{y}(x) I_{1,-}^{1-\alpha} p(x){ }^{C} D_{0,+}^{\alpha} y(x)\right|_{0} \cdot \\
& +\left.y(x) I_{1,-}^{1-\alpha} p(x){ }^{C} D_{0,+}^{\alpha} \bar{y}(x)\right|_{0} \\
& +\left(q(x)-\frac{v^{2}-1 / 4}{x^{2}}\right) y(x) \\
& =-\left.y(x) I_{1,-}^{1-\alpha} p(x){ }^{C} D_{0,+}^{\alpha} \bar{y}(x)\right|_{1} \\
& +
\end{aligned}
$$

By virtue of the boundary conditions (23), (25), we find

$$
(\lambda-\bar{\lambda}) \int_{0}^{1} w_{\alpha}(x)|y(x)|^{2} d x=0
$$

Because $y$ is a nontrivial solution and $w_{\alpha}(x)>0$, it is easily seen that $\lambda=\bar{\lambda}$. The eigenvalues are real.

Theorem 8. The eigenfunctions corresponding with distinct eigenvalues of fractional Bessel operator (15)-(16) are orthogonal weight function $w_{\alpha}$ on $(0,1]$; that is,

$$
\int_{0}^{1} w_{\alpha}(x) y_{\lambda_{1}}(x) y_{\lambda_{2}}(x) d x=0, \quad \lambda_{1} \neq \lambda_{2}
$$


Proof. We have by assumptions fractional Sturm-Liouville operator for Bessel type fulfilled by two different eigenvalues $\left(\lambda_{1}, \lambda_{2}\right)$ and the respective eigenfunctions $\left(y_{\lambda_{1}}, y_{\lambda_{2}}\right)$ :

$$
\begin{gathered}
\mathscr{L}_{\alpha[B]} y_{\lambda_{1}}(x)+\lambda_{1} w_{\alpha}(x) y_{\lambda_{1}}(x)=0, \\
y_{\lambda_{1}}(x)=0, \\
d_{1} y_{\lambda_{1}}(1)+d_{2} I_{1,-}^{1-\alpha} p(1){ }^{C} D_{0,+}^{\alpha} y_{\lambda_{1}}(1)=0, \\
\mathscr{L}_{\alpha[B]} y_{\lambda_{2}}(x)+\lambda_{2} w_{\alpha}(x) y_{\lambda_{2}}(x)=0, \\
y_{\lambda_{2}}(x)=0, \\
d_{1} y_{\lambda_{2}}(1)+d_{2} I_{1,-}^{1-\alpha} p(1){ }^{C} D_{0,+}^{\alpha} y_{\lambda_{2}}(1)=0 .
\end{gathered}
$$

We multiply (30) by function $y_{\lambda_{2}}$ and (32) by function $y_{\lambda_{1}}$, respectively, and subtract.

$$
\left(\lambda_{1}-\lambda_{2}\right) w_{\alpha}(x) y_{\lambda_{1}} y_{\lambda_{2}}=y_{\lambda_{1}} \mathscr{L}_{\alpha[B]} y_{\lambda_{2}}-y_{\lambda_{2}} \mathscr{L}_{\alpha[B]} y_{\lambda_{1}} .
$$

Integrating over interval $(0,1]$ and applying relation (21) we note that the right-hand side of the integrated equality contains only boundary terms:

$$
\begin{aligned}
& \left(\lambda_{1}-\lambda_{2}\right) \int_{0}^{1} w_{\alpha}(x) y_{\lambda_{1}}(x) y_{\lambda_{2}}(x) d x \\
& =\int_{0}^{1} y_{\lambda_{1}}(x) \mathscr{L}_{\alpha[B]} y_{\lambda_{2}}(x) d x \\
& -\int_{0}^{1} y_{\lambda_{2}}(x) \mathscr{L}_{\alpha[B]} y_{\lambda_{1}}(x) d x \\
& =\int_{0}^{1} y_{\lambda_{1}}(x)\left[D_{1,-}^{\alpha} p(x){ }^{C} D_{0,+}^{\alpha} y_{\lambda_{2}}(x)\right. \\
& \left.+\left(q(x)-\frac{v^{2}-1 / 4}{x^{2}}\right) y_{\lambda_{2}}(x)\right] d x \\
& -\int_{0}^{1} y_{\lambda_{2}}(x)\left[D_{1,-}^{\alpha} p(x){ }^{C} D_{0,+}^{\alpha} y_{\lambda_{1}}(x)\right. \\
& \left.+\left(q(x)-\frac{v^{2}-1 / 4}{x^{2}}\right) y_{\lambda_{1}}(x)\right] d x \\
& =-\left.y_{\lambda_{1}}(x) I_{1,-}^{1-\alpha} p(x){ }^{C} D_{0,+}^{\alpha} y_{\lambda_{2}}(x)\right|_{1} \\
& +\left.y_{\lambda_{1}}(x) I_{1,-}^{1-\alpha} p(x){ }^{C} D_{0,+}^{\alpha} y_{\lambda_{2}}(x)\right|_{0} \\
& +\left.y_{\lambda_{2}}(x) I_{1,-}^{1-\alpha} p(x){ }^{C} D_{0,+}^{\alpha} y_{\lambda_{1}}(x)\right|_{1} \\
& -\left.y_{\lambda_{2}}(x) I_{1,-}^{1-\alpha} p(x){ }^{C} D_{0,+}^{\alpha} y_{\lambda_{1}}(x)\right|_{0} \text {. }
\end{aligned}
$$

Using the boundary conditions (31), (33), we obtain that

$$
\left(\lambda_{1}-\lambda_{2}\right) \int_{0}^{1} w_{\alpha}(x) y_{\lambda_{1}}(x) y_{\lambda_{2}}(x) d x=0,
$$

where $\lambda_{1} \neq \lambda_{2}$. Then, the eigenfunctions are orthogonal of this operator.

Remark 9. Let us now give certain auxiliary functions. Because we use the functions, the first of them is as follows:

$$
\begin{aligned}
& I_{0,+}^{\alpha} \frac{(1-x)^{\alpha-1}}{\Gamma(\alpha)} \\
& =(1-0)^{\alpha-1}(x-0)^{\alpha}{ }_{1} \Psi_{2}\left[\begin{array}{cc}
(1,1) & \\
(\alpha,-1) & (\alpha+1,1)
\end{array} \mid-\frac{x-0}{1-0}\right],
\end{aligned}
$$

where ${ }_{1} \Psi_{2}$ is the Fox-Wright function [14]:

$$
\begin{aligned}
{ }_{1} \Psi_{2}\left[\begin{array}{cc}
\left(a_{1}, \alpha_{1}\right) \\
\left(b_{1}, \beta_{1}\right) & \left(b_{2}, \beta_{2}\right)
\end{array} \mid z\right] \\
=\sum_{k=0}^{\infty} \frac{\Gamma\left(a_{1}+\alpha_{1} k\right)}{\Gamma\left(b_{1}+\beta_{1} k\right) \Gamma\left(b_{2}+\beta_{2} k\right)} \frac{z^{k}}{k !} .
\end{aligned}
$$

The properties of the function are determined by the parameters

$$
\begin{gathered}
\Delta=\beta_{1}+\beta_{2}-\alpha_{1}=-1, \\
\delta=\left|\alpha_{1}\right|^{-\alpha_{1}}\left|\beta_{1}\right|^{\beta_{1}}\left|\beta_{2}\right|^{\beta_{2}}=1, \\
\mu=b_{1}+b_{2}-\alpha_{1}+\frac{1-2}{2}=2 \alpha-\frac{1}{2} .
\end{gathered}
$$

Considering Theorem 4 , we note that this function is continuous in $(0,1]$ when order $\alpha>1 / 2$, that is, $\mu>1 / 2$. For $0<\alpha \leq 1 / 2$; it is discontinuous at end $x=1$. The explicitly calculated function allows to estimate the second component of stationary function $\phi_{0}$ of the differential part of SturmLiouville operator

$$
D_{1,-}^{\alpha} p(x){ }^{C} D_{0,+}^{\alpha} \phi_{0}(x)=0
$$

which looks as follows:

$$
\phi_{0}(x)=\xi_{1}+\xi_{2} I_{0,+}^{\alpha} \frac{(1-x)^{\alpha-1}}{\Gamma(\alpha) p(x)}=\xi_{1}+\xi_{2} \psi(\alpha, 0, x) .
$$

The next function is the following integral:

$$
\begin{aligned}
& \varphi(x)=I_{0,+}^{\alpha} I_{1,-}^{\alpha} 1=I_{0,+}^{\alpha} \frac{(1-x)^{\alpha}}{\Gamma(\alpha+1)} \\
& =(1-0)^{\alpha}(x-0)^{\alpha} \\
& \times{ }_{1} \Psi_{2}\left[\begin{array}{c|c}
(1,1) & \\
(\alpha+1,-1) & (\alpha+1,1)
\end{array} \mid-\frac{x-0}{1-0}\right] .
\end{aligned}
$$

Again, using Theorem 4 and calculating parameters according to (39),

$$
\Delta=-1, \quad \delta=1, \quad \mu=2 \alpha+\frac{1}{2} .
$$


Finally,

$$
\alpha>0 \Longrightarrow \mu>\frac{1}{2}
$$

and the obtained Fox-Wright function (42) is continuous in interval $(0,1]$ for any positive order $\alpha$.

Theorem 10. Let $\alpha>1 / 2, x \in(0,1]$ and define

$$
\begin{gathered}
Y_{\lambda}(y)=\left(q(x)-\frac{v^{2}-1 / 4}{x^{2}}\right) y_{\lambda}(x)+\lambda w_{\alpha} y_{\lambda}(x), \\
\tilde{\Delta}=d_{2}+d_{1} \psi(\alpha, 0,1) .
\end{gathered}
$$

Assume that $\widetilde{\Delta} \neq 0$. Then, (15)-(16) are equivalent to the integral equation

$$
\begin{aligned}
& y_{\lambda}(x) \\
& =-I_{0,+}^{\alpha} \frac{1}{p(x)} I_{1,-}^{\alpha} Y_{\lambda}(y)+\left.A(x)\left(I_{0,+}^{\alpha} \frac{1}{p(x)} I_{1,-}^{\alpha} Y_{\lambda}(y)\right)\right|_{x=1},
\end{aligned}
$$

where the coefficient $A(x)$ is

$$
A(x)=\frac{d_{1}}{\widetilde{\Delta}} \psi(\alpha, 0, x)
$$

and functions $\psi$ are defined in (41).

Proof. By means of composition rules, (15) can be rewritten as follows:

$$
D_{1,-}^{\alpha} p(x){ }^{C} D_{0,+}^{\alpha}\left[y_{\lambda}(x)+I_{0,+}^{\alpha} \frac{1}{p(x)} I_{1,-}^{\alpha} Y_{\lambda}(y)\right]=0 .
$$

The last equality suggests that is a stationary function of fractional singular Sturm-Liouville problem for Bessel operator. $D_{1,-}^{\alpha} p(x){ }^{C} D_{0,+}^{\alpha}$ which according to (41) can be found as

$$
\phi_{0}=\xi_{1}+\xi_{2} I_{0,+}^{\alpha} \frac{(1-x)^{\alpha-1}}{\Gamma(\alpha) p(x)}=\xi_{1}+\xi_{2} \psi(\alpha, 0, x) .
$$

Equation (15) in the form of

$$
y_{\lambda}(x)+I_{0,+}^{\alpha} \frac{1}{p(x)} I_{1,-}^{\alpha} Y_{\lambda}(y)=\xi_{1}+\xi_{2} \psi(\alpha, 0, x)
$$

proves we should connect coefficients $\xi_{j}$ values $d_{j}, j=1,2$ determining the boundary conditions (16).

Let us note that the following formula results from composition rules (11) and (50):

$$
I_{1,-}^{1-\alpha} p(x){ }^{C} D_{0,+}^{\alpha} y_{\lambda}(x)=-I_{1,-}^{1} Y_{\lambda}(y)+\xi_{2} .
$$

For continuous function $y_{\lambda}$, we obtain the following values as the ends

$$
\begin{gathered}
\left.I_{1,-}^{1-\alpha} p(x){ }^{C} D_{0,+}^{\alpha} y_{\lambda}(x)\right|_{x=0}=-\int_{0}^{\pi} Y_{\lambda}(y)+\xi_{2}, \\
\left.I_{1,-}^{1-\alpha} p(x){ }^{C} D_{0,+}^{\alpha} y_{\lambda}(x)\right|_{x=1}=\xi_{2}
\end{gathered}
$$

respectively, for $y_{\lambda}$. Using (50), we find

$$
\begin{gathered}
y_{\lambda}(0)=\phi_{0}(0)=\xi_{1}, \\
y_{\lambda}(1)=\phi_{0}(1)-\left.I_{0,+}^{\alpha} \frac{1}{p(x)} I_{1,-}^{\alpha} Y_{\lambda}(y)\right|_{x=1} \\
=\xi_{1}+\xi_{2} \psi(\alpha, 0,1)-\left.I_{0,+}^{\alpha} \frac{1}{p(x)} I_{1,-}^{\alpha} Y_{\lambda}(y)\right|_{x=1} .
\end{gathered}
$$

The following set of linear equations for coefficients $\xi_{j}$ results from (52)-(54)

$$
\begin{gathered}
\xi_{1}=0 \\
d_{1} \xi_{1}+\xi_{2}\left(d_{2}+d_{1} \psi(\alpha, 0,1)\right)=d_{1} F
\end{gathered}
$$

where $F=\left.I_{0,+}^{\alpha}(1 / p(x)) I_{1,-}^{\alpha} Y_{\lambda}(y)\right|_{x=1}$.

Since $\widetilde{\Delta} \neq 0$, the solution for coefficients $\xi_{j}(j=1,2)$ is unique:

$$
\begin{gathered}
\xi_{1}=0, \\
\xi_{2}=\frac{d_{1} F}{\widetilde{\Delta}},
\end{gathered}
$$

Substituting the previous solution into (50) we recover the equivalent integral equation (46).

Furthermore, we give notation such as

$$
\begin{gathered}
m_{p}=\min _{x \in[0,1]}|p(x)|, \\
A=\|A(x)\|, \quad M_{\varphi}=\|\varphi(x)\| .
\end{gathered}
$$

The proof is completed.

\section{Conclusion}

In the paper, we have extended the scope of some spectral properties of singular fractional Sturm-Liouville problem. We pointed that its eigenvalues related to the Bessel operator with the certain boundary conditions are real and its eigenfunctions corresponding to distinct eigenvalues are orthogonal. Furthermore, we showed that fractional Bessel operator is self-adjoint. Spectral properties of Sturm-Liouville theory are applied to the fractional theory. Our results are important in point of the fractional Sturm-Liouville theory.

\section{Acknowledgments}

The author sincerely thanks the editor and the reviewer for their valuable suggestions and comments.

\section{References}

[1] R. S. Johnson, An Introduction To Sturm-Liouville Theory, University of Newcastle, 2006.

[2] A. Zettl, Sturm-Liouville Theory, vol. 121 of Mathematical Surveys and Monographs, American Mathematical Society, Providence, RI, USA, 2005. 
[3] W. O. Amrein, A. M. Hinz, and D. B. Pearson, Eds., SturmLiouville Theory, Past and Present, Birkhäuser, Basel, Switzerland, 2005.

[4] E. S. Panakhov and R. Yilmazer, "A Hochstadt-Lieberman theorem for the hydrogen atom equation," Applied and Computational Mathematics, vol. 11, no. 1, pp. 74-80, 2012.

[5] B. M. Levitan and I. S. Sargsjan, Introduction to Spectral Theory: Self adjoint Ordinary Differential Operators, American Mathematical Society, Providence, RI, USA, 1975.

[6] J. Qi and S. Chen, "Eigenvalue problems of the model from nonlocal continuum mechanics," Journal of Mathematical Physics, vol. 52, no. 7, Article ID 073516, 2011.

[7] E. S. Panakhov and M. Sat, "Reconstruction of potential function for Sturm-Liouville operator with Coulomb potential," Boundary Value Problems, vol. 2013, article 49, 2013.

[8] A. Carpinteri and F. Mainardi, Eds., Fractals and Fractional Calculus in Continum Mechanics, Telos: Springer, 1998.

[9] B. J. West, M. Bologna, and P. Grigolini, Physics of Fractal Operators, Springer, New York, NY, USA, 2003.

[10] I. Podlubny, Fractional Differential Equations, vol. 198, Academic Press, San Diego, Calif, USA, 1999.

[11] R. Hilfer, Ed., Applications of Fractional Calculus in Physics, World Scientific, Singapore, 2000.

[12] S. G. Samko, A. A. Kilbas, and O. I. Marichev, Fractional Integrals and Derivatives: Theory and Applications, Gordon and Breach, Philadelphia, Pa, USA, 1993.

[13] K. S. Miller and B. Ross, An Introduction to the Fractional Calculus and Fractional Differential Equations, John Wiley \& Sons, New York, NY, USA, 1993.

[14] A. A. Kilbas, H. M. Srivastava, and J. J. Trujillo, Theory and Applications of Fractional Differential Equations, vol. 204, Elsevier, Amsterdam, The Netherlands, 2006.

[15] R. Yilmazer and E. Bas, "Fractional solutions of confluent hypergeometric equation," Journal of the Chungcheong Mathematical Society, vol. 25, no. 2, pp. 149-157, 2012.

[16] X. Jiang and H. Qi, “Thermal wave model of bioheat transfer with modified Riemann-Liouville fractional derivative," Journal of Physics A, vol. 45, no. 48, Article ID 485101, 2012.

[17] X. Jiang and $\mathrm{M}$. Xu, "The time fractional heat conduction equation in the general orthogonal curvilinear coordinate and the cylindrical coordinate systems," Physica A, vol. 389, no. 17, pp. 3368-3374, 2010.

[18] E. Nakai and G. Sadasue, "Martingale Morrey-Campanato spaces and fractional integrals," Journal of Function Spaces and Applications, vol. 2012, Article ID 673929, 29 pages, 2012.

[19] Y. Wang, L. Liu, and Y. Wu, "Existence and uniqueness of a positive solution to singular fractional differential equations," Boundary Value Problems, vol. 2012, article 81, 2012.

[20] D. Băleanu and O. G. Mustafa, "On the existence interval for the initial value problem of a fractional differential equation," Hacettepe Journal of Mathematics and Statistics, vol. 40, no. 4, pp. 581-587, 2011.

[21] M. Klimek, On Solutions of Linear Fractional Differential Equations of a Variational Type, The Publishing Office of Czestochowa, University of Technology, Czestochowa, Poland, 2009.

[22] Q. M. Al-Mdallal, "An efficient method for solving fractional Sturm-Liouville problems," Chaos, Solitons and Fractals, vol. 40, no. 1, pp. 183-189, 2009.
[23] V. S. Ertürk, "Computing eigenelements of Sturm-Liouville problems of fractional order via fractional differential transform method," Mathematical \& Computational Applications, vol. 16, no. 3, pp. 712-720, 2011.

[24] M. Klimek and O. P. Argawal, "On a regular fractional SturmLiouville problem with derivatives of order in $(0,1)$," in Proceedings of the 13th International Carpathian Control Conference, May 2012. 


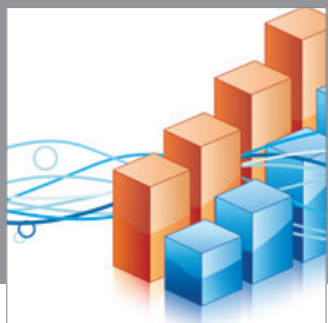

Advances in

Operations Research

mansans

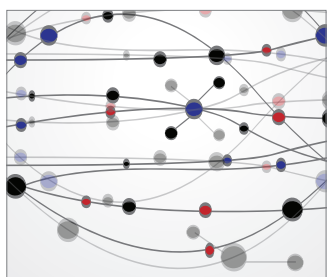

The Scientific World Journal
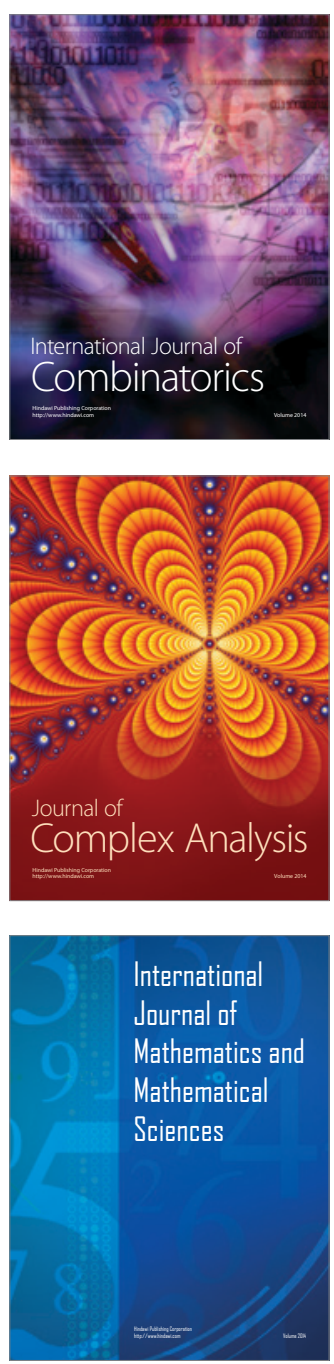
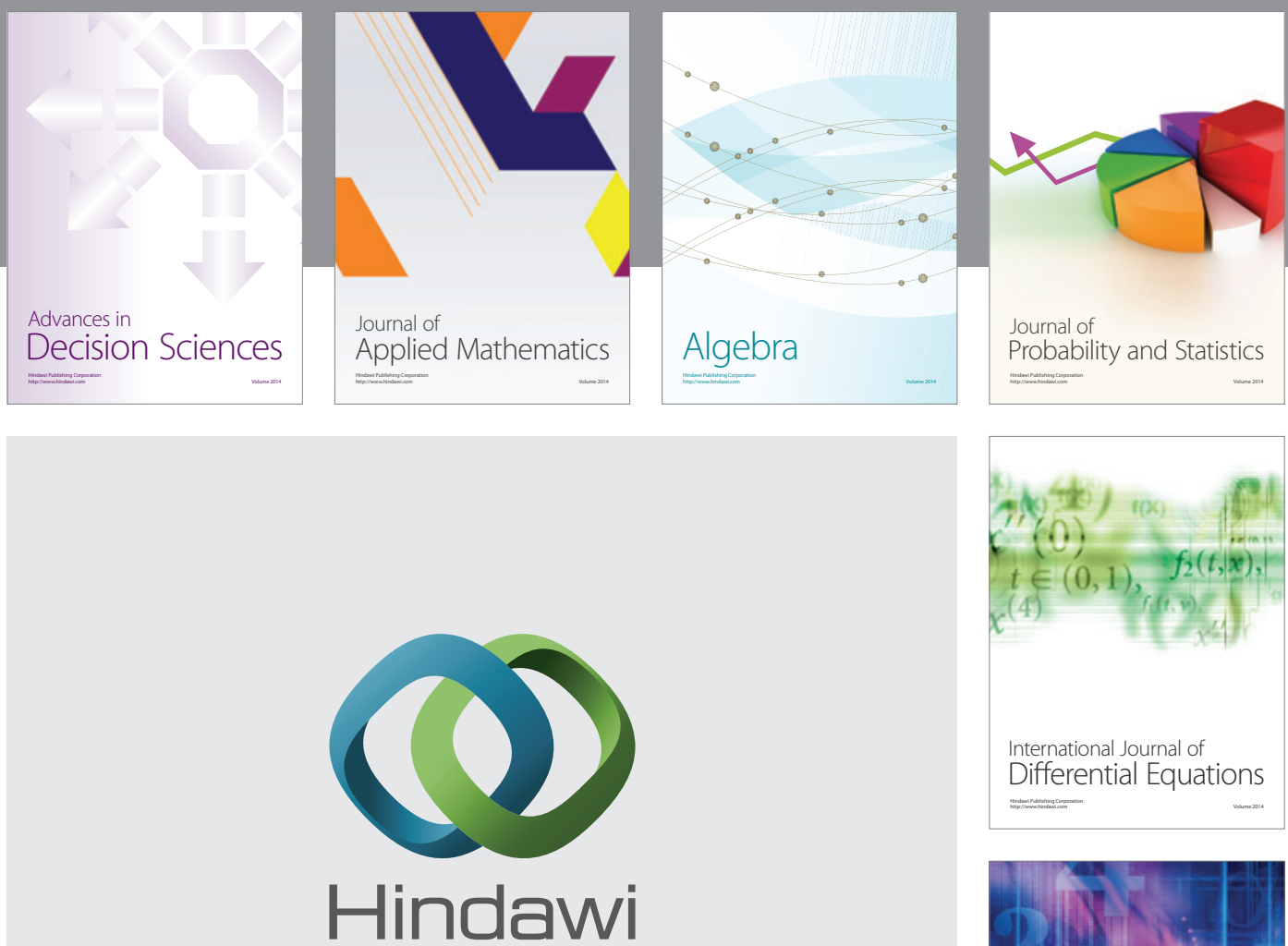

Submit your manuscripts at http://www.hindawi.com
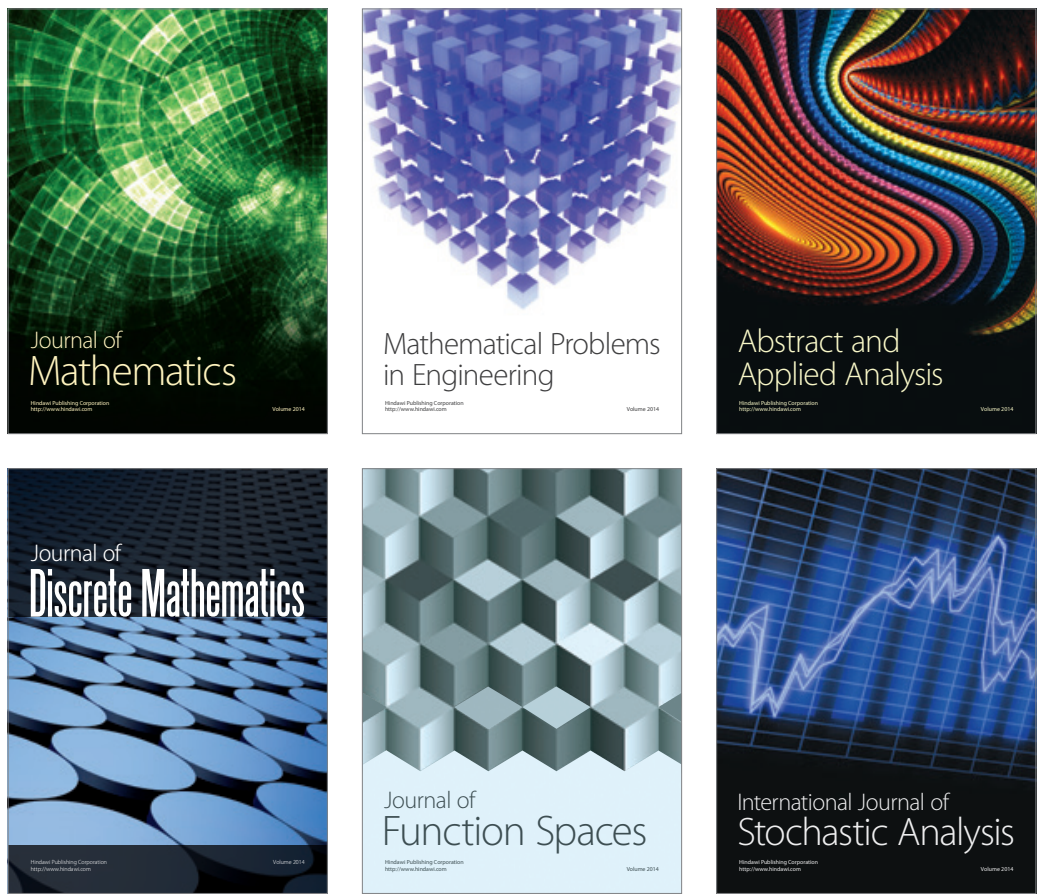

Journal of

Function Spaces

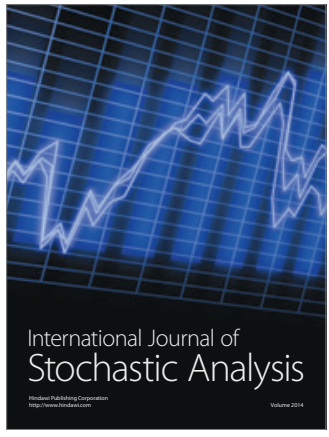

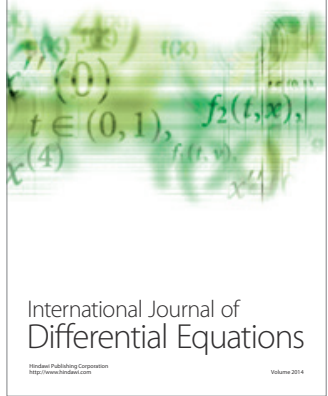
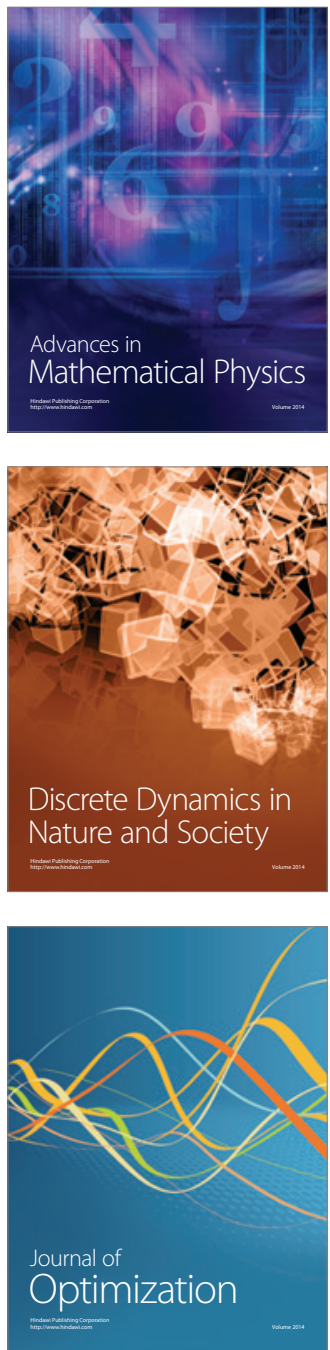